\title{
Sex differences in learning from exploration
}

+to whom correspondence should be addressed:

8 Nicola Grissom

9 Department of Psychology

10 University of Minnesota

1175 East River Rd

12 Minneapolis, MN 55455

13 ngrissom@umn.edu

14

15 Becket Ebitz,

16 Department of Neuroscience,

17 Faculty of Medicine

18 Université de Montréal

19 2960, chemin de la Tour, local 111

20 Montréal, QC H3T 1J4 Canada

21 becket@ebitzlab.com 


\section{Abstract}

24 Sex-based modulation of cognitive processes could set the stage for individual

25 differences in vulnerability to neuropsychiatric disorders. While value-based decision making

26 processes in particular have been proposed to be influenced by sex differences, the overall

27 correct performance in decision making tasks often show variable or minimal differences across

28 sexes. Computational tools allow us to uncover latent variables that define different decision

29 making approaches, even in animals with similar correct performance. Here, we quantify sex

30 differences in mice in the latent variables underlying behavior in a classic value-based decision

31 making task: a restless 2-armed bandit. While male and female mice had similar accuracy, they

32 achieved this performance via different patterns of exploration. Male mice tended to make more

33 exploratory choices overall, largely because they appeared to get "stuck" in exploration once

34 they had started. Female mice tended to explore less, but learned more quickly during

35 exploration. Together, these results suggest that sex exerts stronger influences on decision

36 making during periods of learning and exploration than during stable choices. Exploration during

37 decision making is altered in people diagnosed addictions, depression, and neurodevelopmental

38 disabilities, pinpointing the neural mechanisms of exploration as a highly translational avenue

39 for conferring sex-modulated vulnerability to neuropsychiatric diagnoses.

41 Keywords: sex differences, exploration, exploration-exploitation tradeoff, reinforcement

42 learning, decision making, animal models 


\section{Introduction}

Essentially every neuropsychiatric condition shows sex and/or gender biases in risk, presentation, etiology, and prognosis (Green et al., 2019; Grissom and Reyes, 2018; Shansky, 2019). This raises the possibility that sex-modulated biological mechanisms could modulate cognitive processes that confer vulnerability and/or resilience to mental health challenges. However, sex differences in cognitive task performance can be difficult to detect and even more variable than would be expected given the non-dichotomous, overlapping impacts of sex mechanisms on cognition (Maney, 2016). An underrecognized source of variability in cognitive tasks is that there can be multiple ways to achieve the same level of performance on the primary dependent variables used to assess these tasks, such as "number of correct responses". This means that equivalent levels of performance could mask individual differences in how males and females are solving the same problem. Indeed, we have recently shown that examining the latent strategies underlying task performance --rather than differences in final performance--can reveal that individual males and females can take very different strategic paths to the learning of actionoutcome associations (Chen et al., 2020). Here, we applied computational tools to characterize sex differences in the latent variables underlying behavior to understand sex differences in a key cognitive process regulating reward-guided behaviors: balancing exploration and exploitation.

In an uncertain world, we must balance two goals: exploiting rewarding options when they are available, but also exploring alternatives that could be more rewarding or provide new information about the world. Too little exploration makes behavior inflexible and perseverative. Too much makes it impossible to sustain rewarding behaviors. Exploration is dysregulated in numerous neuropsychiatric disorders (Addicott et al., 2017; Wilson et al., 2021), many of which are also sex-biased (Green et al., 2019; Grissom and Reyes, 2018; Shansky, 2019). This suggests that sex differences in exploration and exploitation could contribute to sex-linked vulnerability to these conditions, though we do not yet understand how exploration and exploitation differ with sex. Because exploration is a major source of errors in task performance more broadly (Ebitz et al., 2019; Pisupati et al., 2019), sex-differences in exploration may influence behavior in complex ways in tasks which are not intended to directly measure this tendency and could contribute to performance differences and variability seen across tasks and species (Grissom and Reyes, 2018; van den Bos et al., 2013). 
To examine whether there are sex differences in exploration, we trained male and female mice on a classic explore/exploit task, a spatial restless two-armed bandit (Ebitz et al., 2018).

75 Males showed higher levels of exploration than females throughout the task. This was because

76 males were more likely to get "stuck" in extended periods of exploration before committing to a

77 favored choice. On the other hand, females showed elevated reward learning specifically during bouts of exploration, making exploratory trials more informative, which allowed them to start exploiting a favored choice earlier than males. Together, these results demonstrate that while the overall performance was similar, males and females exhibited different patterns of exploration while interacting with the same uncertain environment.

\section{Results}

Age-matched male and female wildtype mice $(\mathrm{n}=32,16$ per sex, strain B6129SF1/J) were trained to perform a restless two-armed spatial bandit task in touch-screen operant chambers (Figure 1A). Animals were presented with two physically identical targets (squares) on the left and right of the screen each trial and indicated their choices by nose poking at one of two target locations. Each location offered some probability of reward, which changed slowly and randomly across trials, and independently across targets. The dynamic reward contingencies encouraged the animals to constantly balance exploration and exploitation. The animals had to exploit a good option when it is found, but also occasionally explore the other option, whose

92 drifting values meant that it could become better at any time. Mice performed 2 repetitions of 4

93 consecutive sessions of the restless bandit task, measuring 8 sessions total. Each session

94 consisted of 300 trials.

To examine task performance, we first calculated the average probability of reward obtained in males and females. Because rewards schedules were stochastic, sessions could differ

97 slightly in the amount of reward that was available. We therefore compared performance against 98 the average probability of reward if choosing randomly within each session. Regardless of sex, 99 mice were able to earn reward more frequently than chance (Figure 1B, two-way ANOVA, F(1, $10060)=228.9, \mathrm{p}<0.0001)$. There was no significant sex difference in the probability of rewards 101 acquired above chance (main effect of sex, $\mathrm{F}(1,30)=0.05, \mathrm{p}=0.83$ ). 
102

103

104

105

106

107

108

109

110

Similar levels of accuracy do not require or imply a similar approach to the task. Our previous study suggested that males and females could achieve similar learning performance via divergent decision making strategies (Chen et al., 2020). However, different strategies might take different amounts of time to execute (Chen et al., 2020; Filipowicz et al., 2019; Kool et al., 2010; Kurdi et al., 2019). Therefore, if males and females had adopted strategies here, then we might expect response time to systematically differ between males and females, despite the similarities in learning performance. Indeed, females responded significantly faster than did males (Figure 1C, main effect of sex, $\mathrm{t}(30)=3.52, \mathrm{p}=0.0014)$, suggesting that decision making computations may differ across sexes and, if so, that the strategies that tended to be used by females were faster than those used by males.

\section{A Hidden Markov model (HMM) identifies distinct features of exploratory and exploitative choices in mice.}

Despite similar performance, response time differences suggested that males and females employed different strategies in this task. One possible difference was sex differences in the level of exploration. Prior research has shown that exploratory choices take longer than exploitative choices (Ebitz et al., 2019, 2018). Therefore, perhaps males took longer to make a choice because a greater proportion of their choices were exploratory. To test this hypothesis, we used a hidden Markov model (HMM) to determine whether choices were exploratory or exploitative (Ebitz et al., 2018).

The HMM allows us to make statistical inferences about the probability that each choice was due to exploration or exploitation via modeling these as the latent goal states underlying choice (see Methods). Because this approach to inferring exploration is agnostic to the generative computations and depends only on the temporal statistics of choices (Ebitz et al., 2020, 2019, 2018), it is particularly ideal for circumstances like this one, where we suspect that the generative computations may differ across groups.

The HMM method has never before been used to quantify exploration in mice, so we first asked whether it was appropriate here. The HMM method works because sequences of exploratory decisions look very different from exploitative ones, at least in reinforcement learning agents and primates (Ebitz et al., 2018). When agents exploit, they repeatedly sample 
131 the same option, switching only very rarely. However, because exploration requires investigatory

132 samples, runs of exploratory choices tend not to repeat the same option. They tend to switch far

133 more frequently, closer to what we would expect from random samples from the environment

134 (Ebitz et al., 2018). Therefore, if mice were alternating between exploration and exploitation in

135 this task, we would expect to see evidence of two distinct patterns of switching in their behavior.

136 Indeed, choice run durations (i.e. the distribution of inter-switch intervals) were parsimoniously

137 described as a mixture of two different patterns: one regime where choices switched quickly

138 (mean switching time $=1.7$ trials, compared to random choices at 2 trials; $80 \%$ of choice runs)

139 and one regime where they changed slowly (mean switching time $=6.8$ trials; Supplemental

140 Figure 1A). Thus, mice had evidence of fast-switching (putatively explore) and slow-switching

141 (putatively exploit) regimes in their behavior. Note that explore-labeled choices are more likely

142 to also be switch choices, but not all explore-labeled choices are switches, and not all exploit-

143 labeled choices are stay decisions.

144 Next, we used a simple 2-parameter HMM to infer the likelihood that each choice was

145 part of the exploratory regime, or the exploitative one (see Methods). To evaluate the face

146 validity of the HMM labels, we asked whether HMM-labeled exploratory choices matched the

147 normative definition of the term. First, by definition, exploration is a pattern of non-reward-

148 maximizing choices whose purpose is learning about rewards. This means exploratory choices

149 should be (1) orthogonal to reward value, and (2) exhibit enhanced reward learning.

Explore-labeled choices were non-reward-maximizing: they were orthogonal to reward

151 value (Supplemental Figure 1B; the average value of choices chosen during exploration was

152 not different from chance; one sample t-test, $\mathrm{t}(10)=0.16, \mathrm{p}=0.87$ ). Reward learning was also

153 elevated during exploration. During HMM-labeled exploratory states, the outcome of choices

154 had more influence on the subsequent decision - animals were more likely to stay with the same

155 choice if rewarded and switch if not rewarded (Supplemental Figure 1C, two-way RM

156 ANOVA, interaction term, $\mathrm{F}(1,31)=51.2, \mathrm{p}<0.001)$.

Finally, we asked whether the labels inferred by the HMM produced differences in

158 behavioral metrics other than choices. We computed average choice response time separately for

159 exploratory and exploitative states. The results suggested that choice response time was

160 significantly longer during exploration than exploitation (Supplemental Figure 1D, t-test, t(31) 
$161=3.66, \mathrm{p}=0.0009$ ), which is consistent with previous findings that exploration slows down

162 decision making (Ebitz et al., 2018). To eliminate the possibility that exploration was merely

163 disengagement from the task, we examined average reward retrieval time during exploratory and

164 exploitative states. There was no significant difference in reward retrieval time between two

165 states (Supplemental Figure 1E, t-test, $\mathrm{t}(31)=0.05, \mathrm{p}=0.95$ ), suggesting that animals were not

166 more disengaged from the task during exploration than exploitation. They were only slower in

167 making a decision. Together, these results demonstrated that HMM-labeled exploration was

168 meaningful, non-reward-maximizing, and accompanied by enhanced reward learning, matching

169 the normative definition of exploration.

170 Males made more exploratory choices than females, because they explored for longer 171 periods of time once they started.

172 With more confidence in the validity of HMM-inferred states, we found that males, on 173 average, were more likely to be in the exploratory regime than the exploitative one, with $72.9 \%$

$174 \pm 11.5 \%$ STD of trials labeled as exploratory (Figure 1D). Females explored much less with

175 only $55.4 \% \pm 20.4 \%$ STD of trials being exploratory (Figure 1E, t-test, $\mathrm{t}(30)=2.98, \mathrm{p}=0.0056$;

$17695 \% \mathrm{CI}$ for the difference between the sexes $=[5.5 \%, 29.3 \%])$. As groups, males and females

177 were reasonably, but not perfectly discriminable in terms of the proportion of exploratory

178 choices (Figure 1F, receiver operating characteristic analysis, AUC $=0.76 \pm 0.09,95 \%$ CI for

179 the difference $=[0.59,0.93], \mathrm{p}=0.013)$. These differences were largely driven by the greater

180 male tendency to keep exploring once they started. Males repeated exploration $92.1 \%( \pm 3.4 \%$

181 STD) of the time, while females stopped exploring and committed to a choice more quickly,

182 repeating exploration only $83.1 \%( \pm 16.8 \%$ STD) of the time ( $\mathrm{t}$-test, $\mathrm{t}(30)=2.09, \mathrm{p}=0.045,95 \%$

$183 \mathrm{CI}$ for the difference $=[0.2 \%, 17.8 \%])$. There were no significant differences in the other

184 parameter of the HMM (probability of repeating exploitation: males $=83.5 \pm 3.7 \%$; females $=$

$18579.7 \pm 22.1 \% ; \mathrm{t}(30)=0.69, \mathrm{p}=0.5)$.

Although sex differences in model parameters were modest, analyzing the full dynamics

187 of the fitted HMMs again supported a robust sex difference in the tendency to explore (see

188 Methods). In models fit to males, exploration was a deeper, more "sticky" behavioral state

189 (Figure 1G, stationary probability of exploration $=68.0 \pm 8.5 \%$ STD), compared to models fit to

190 females, where exploration and exploitation were more closely matched (54.4 $\pm 18.4 \%$ STD; 
191 different from males: $\mathrm{t}(30)=2.68, \mathrm{p}=0.012,95 \% \mathrm{CI}$ for the difference $=[3.2 \%, 23.9 \%])$. This

192 suggests that males were more likely to get "stuck" in an extended exploratory period, requiring

193 more energy to escape from exploring and start exploiting a good choice.

194 Multiple variables in reinforcement learning models may be the cause of increased 195 exploration.

The results from the HMM analyses suggest that males were, on average, more exploratory than females, and not because they were more likely to initiate exploration, but because they were more likely to become "stuck" in exploration. This suggests that there were sex differences in the animals' approach to this task. However, a crucial question remained unanswered: what computational differences made the males more exploratory? To address this question, we turned to reinforcement learning models to look for individual variability in latent cognitive parameters that could influence exploration and exploitation (Daw et al., 2006; Ishii et al., 2002; Jepma and Nieuwenhuis, 2011; Pearson et al., 2009).

We began by considering a simple, 2-parameter RL model, with one learning rate parameter $(\alpha)$ and one parameter for decision noise (inverse temperature $\beta$ ). Traditionally, only the latter parameter is thought to be related to exploration, so most previous studies of exploration have focused exclusively on this inverse temperature parameter (Beeler et al., 2010; Cinotti et al., 2019). However, we realized that exploration in an RL model is a function of both the difference in subjective values and the decision-noise (Figure 2A). This implied that there could be multiple ways to increase exploration even in this simple 2-parameter RL model.

Indeed, changing the inverse temperature of the Softmax function influences the 212 probability of exploration - the larger value of $\beta$, the lower the decision noise, thus lower

213 probability of exploration. Consistent with this prior work, we found that changing beta robustly 214 changed the probability of exploration in simulations from the model (Figure 2B; GLM, main

215 effect of inverse temperature, $\beta 1=-0.11, \mathrm{p}<0.0001)$. The second latent parameter, the learning

216 rate, could also mediate the level of exploration by changing the value difference between

217 options. The learning rate dictates the rate of value updating from each outcome. For example, 218 when an agent receives two consecutive rewards (Figure 2A (bottom)), a higher learning rate 219 expands the cumulative value difference between the rewarded chosen option and the unchosen 
220 option, increasing the probability of exploiting the higher value option. The result of computer

221 simulation confirmed this theory that learning rate also influences the probability of exploration

222 (Figure 2B; GLM, main effect of learning rate, $\beta 2=-0.10$, $p<0.0001$ ). Moreover, there were

223 some values for alpha at which changing decision noise had no effect on exploration whatsoever

224 - alpha and beta interact to influence exploration (Figure 2C; GLM, interaction term, $\beta 3=-0.38$,

$225 \mathrm{p}<0.0001)$. This was because when the learning rate is very low, exploration does not provide

226 as much information about the value of the options, thus it takes longer to learn about which

227 option is better. As a result, exploration is prolonged (duration of an exploration periods) and

228 increased (the number of exploration bouts). Together, these results suggest that sex-differences

229 in exploration (and perhaps individual differences in exploration more broadly) could be due to

230 multiple different changes in the computations underlying behavior.

231 Females had higher learning rates than males, and showed evidence of meta-learning.

In order to understand the computational basis for the sex difference in exploration, we fit

233 RL models in order to consider a variety of latent variables that could, either alone or in

234 combination, influence animals' exploratory behavior. Our ability to make inferences about

235 changes in model parameters is highly sensitive to the correct specification of the model.

236 Therefore, we first compared the fit of six reinforcement learning (RL) models that could

237 potentially characterize animals" choice behaviors : (1) a "random" model with some overall bias

238 for one choice over the other, (2) a "noisy win stay lose shift" model that assumes a win stay lose

239 shift policy with some level of randomness, (3) a two-parameter "RL" model with a consistent

240 learning rate and some inverse temperature that captures decision noise, (4) a four-parameter

241 "RLCK" model that captures both value-based and value-independent decision with separate

242 parameters for learning rate, decision noise, choice bias, and choice stickiness, (5) a five-

243 parameter "RLCK $\gamma$ " model that incorporates separate learning rate for rewarded and unrewarded

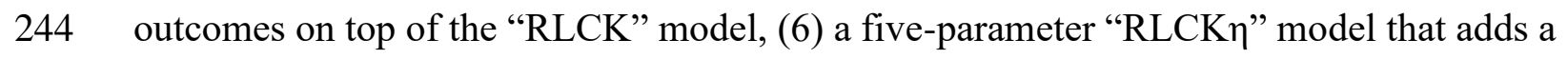

245 parameter that tunes the weight between value-based and choice-based decision to the "RLCK"

246 model (see methods, Figure 3A).

247 Although model fitting was slightly different across sexes, in both males and females, the

248 "RLCK" model, four-parameter model with value and choice kernel updating policies, best

249 characterized animals' choice behaviors in this task among all six models (Figure 3B). To 
confirm that this model was able to capture animals' choice regardless of their learning performance, we used the parameters optimized by the model to simulate choices and compared with the actual choices of animals. We plotted the simulated choices and actual choices against the matching law (Poling et al., 2011), which dictates that the probability of choice is proportional to the probability of reward. The result supported that this four-parameter model was able to characterize animals' choice behaviors regardless of whether the animal was following the matching law (Supplemental Figure 2). This suggests that the best fit model, the

257 four-parameter reinforcement learning - choice kernel (RLCK) model, captured both valuedependent and value-independent choices.

The RLCK model had 4 parameters, which we then compared across sexes. We found that females had significantly higher learning rate $(\alpha)$ than males (Figure $3 \mathbf{C}, \mathrm{t}(30)=2.40, \mathrm{p}=$ $0.02)$ but there was no significant difference across sexes in other parameters $(\beta: t(30)=1.44, p$ $\left.=0.16 ; \alpha_{c}: \mathrm{t}(30)=1.40, \mathrm{p}=0.17 ; \beta_{\mathrm{c}}: \mathrm{t}(30)=1.73, \mathrm{p}=0.09\right)$. To examine whether the higher

263 learning rate in females was driven by a few individuals with extremely high learning rates, we plotted the distribution and calculated the separability of learning rates of two sexes. As groups, males and females were reasonably discriminable in terms of the learning rate (Figure 3D, receiver operating characteristic analysis, $\mathrm{AUC}=0.72 \pm 0.09,95 \% \mathrm{CI}$ for the difference $=[0.54$, 0.90], $\mathrm{p}=0.035$ ). These results suggested that the difference in the level of exploration between

268 males and females was not due to differences in decision noise, but instead due to differences in learning rate.

Furthermore, when compared learning rate across sessions within sex, females, but not

271 males, showed increased learning rate over experience with task (Figure 3E, repeated measures

272 ANOVA, female: main effect of time, $\mathrm{F}(2.26,33.97)=5.27, \mathrm{p}=0.008$; male: main effect of

273 time, $\mathrm{F}(2.5,37.52)=0.23, \mathrm{p}=0.84)$. Females not only learned more quickly but they also

274 "learned to learn", while males did not. This points to sex differences in meta-learning, with

275 females learning not only the immediate choice-outcome association but also the overall

276 dynamic reward structure of the environment (Doya, 2002).

\section{Females learned more during exploratory choices than males.}


The results of HMM model and RL models revealed significant sex differences in exploration, paralleled by sex differences in rate of learning. What remains unclear is how sex, explore-exploit states, and reward outcomes all interact together to influence the animals' choices. Therefore, we conducted a 4-way repeated measures ANOVA to examine how (1) positive and negative outcomes, (2) explore-exploit states, (3) sex, and (4) subject identity (nested in sex) all came together to influence choice: whether animals would repeat their last choice (stay) or try a different option (switch; Table 1). This 4-way repeated measure ANOVA allowed us to understand the main effect of sex, state, and outcome, as well as all pair and tripletwise interaction effects, on how animals learned from previous rewards. Note that in previous analyses, we used subject averaged data but since subject average (16 subjects each sex) is underpowered to detect a 3-way interaction effect, we used session averaged data to increase the power to detect any effects across sex, state and outcome.

The results revealed an expected significant main effect of outcome on stay-switch decisions (main effect of outcome, $\mathrm{p}<0.00001$ ). This effect was driven by the tendency of animals to repeat the previous choice (i.e.: not switching) after obtaining a reward (post hoc t-test compared to chance at 0.50, mean $=0.75,95 \% \mathrm{CI}=[0.74,0.77], \mathrm{t}(255)=34.33, \mathrm{p}<0.0001)$ and a much smaller tendency switch more often than chance after reward omission (mean $=0.52$,

$29595 \% \mathrm{CI}=[0.50,0.55]$, post hoc t-test, $\mathrm{t}(255)=2.05, \mathrm{p}=0.04)$. The tendency to switch or stay also differed by sex, with females more likely to repeat a previous choice and males more likely to switch (main effect of sex, $\mathrm{p}<0.00001$; post-hoc t-test on $\mathrm{p}($ switch): $\mathrm{t}(254)=4.12, \mathrm{p}<0.0001$ ).

298 There was also a significant interaction effect between sex and outcome (sex X reward interaction, $\mathrm{p}<0.00001)$. To understand how reward and reward omission differentially affect choice across sexes, we conducted post-hoc win-stay lose-shift analyses. We found that female mice displayed more win-stay behaviors, indicating that they were more likely than the males to repeat behaviors that produced reward on the previous trial (Figure 4A, sex X reward interaction, $\mathrm{p}<0.000001$, Table 1; post hoc t-test: $\mathrm{t}(254)=5.53, \mathrm{p}<0.0001$ ). As groups, males and females were reasonably, but not perfectly discriminable in terms of the proportion of win stay choices (Figure 4B, receiver operating characteristic analysis, AUC $=0.74 \pm 0.09,95 \% \mathrm{CI}$ for the difference $=[0.56,0.92], p=0.0195)$. In contrast, male mice tended to shift even when

307 the previous choice was rewarded. 
There was no significant sex difference in learning from losses (Figure 4C, $\mathrm{t}(254)=$ $1.40, \mathrm{p}=0.16)$, but this did not mean that sex differences in learning were solely due to sex differences in learning from wins. There were at least two ways that we could observe an equivalent tendency to lose-shift across sexes in this task. One possibility is that females only

312 learn more from positive outcomes, but not negative ones. However, the other possibility is that

313 this lack of a difference in lose-shift behaviors between males and females was an artifact of the

314 tendency of males to explore more frequently. Since males spend more time exploring (Figure

315 1E) and learning from both wins and losses is enhanced during exploration (Supplemental

316 Figure 1D), males could lose-shift less frequently than females during both exploration and

317 exploitation, yet still lose-shift exactly as much as females because a greater proportion of their

318 choices were exploratory.

To dissociate these possibilities, we next examined the effects of exploration and exploitation. The ANOVA revealed a significant main effect of state, resonating with the result of HMM model validation that animals were more likely to switch during exploratory state than during exploitative state (Table 1, main effect of state, $\mathrm{p}<0.00001$; sex $\mathrm{X}$ state interaction, $\mathrm{p}=$ 0.0667; Supplemental Figure 1C). Critically, there was also a significant 3-way interaction between sex, explore/exploit state, and reward (Figure 4E, F, Table 1, sex X reward X state interaction, $\mathrm{p}=0.0438$ ). This could imply sex-linked differences in reward learning across exploration and exploitation. To determine if this was true, we separated out probability of loseshift according to whether it happened during exploration or exploitation, as inferred from the Hidden Markov model. Males switched less after losses than females within exploratory states (Figure 4D; post hoc t-test on session averages: sex difference within exploration: $\mathrm{p}<0.001$, $\mathrm{t}(251)=3.39)$, though there was no significant sex difference within exploitation $(\mathrm{p}=0.06$, $\mathrm{t}(243)=1.87$; note that differing degrees of freedom are due to the fact that exploitation was not observed in some sessions for some animals). This supported the second hypothesis that males

333 lose-shift less than females both when exploring and when exploiting, but that there was no

334 difference in lose-shift overall because males spent more time in a state in which both win-stay and lose-shift choices occur more frequently (Supplemental Figure 1C). We also found that the

336 increased tendency to win-stay that we observed in females was driven by the explore choices

337 (Figure 4E; post-hoc t-test: $\mathrm{p}=0.015, \mathrm{t}(251)=2.55$ ). There was no significant difference in 338 win-stay between males and females during exploit choices (post-hoc t-test: $\mathrm{p}=0.09, \mathrm{t}(244)=$ 
1.68). Together these results suggest that females were better explorers (i.e.: they had increased reward learning during exploration), whereas males learned slower during exploration but compensated this learning disadvantage by exploring more frequently.

These effects were not driven by idiosyncratic strategic differences between the sexes (e.g., shifting only after two losses). We used a model-free approach to quantify the extent to which behavior was structured without making strong assumptions about what form this structure might take. We calculated conditioned mutual information for all sessions across sexes (Leao et al., 2004; Wyner, 1978), to examine how choice behavior was influenced by information of past choice history, given the immediate outcome. The result suggested that mutual information was higher in females than males, suggesting that females were using more information from the past choice and outcome to make their current decision. (Figure 5G, t $(30)$ $=2.65, \mathrm{p}=0.013)$. As groups, males and females were reasonably discriminable in terms of mutual information (Figure 5H, receiver operating characteristic analysis, AUC $=0.74 \pm 0.09$, $95 \% \mathrm{CI}$ for the difference $=[0.57,0.92], \mathrm{p}=0.0195)$. Together, these results reinforced our conclusion that females were learning more: utilizing more information from the past trial to make current choices.

\section{Discussion}

Sex mechanisms biasing the preferred approaches taken during cognitive tasks are potentially significant contributors to task performance. We used a combination of computational modeling approaches to characterize sex differences in a canonical explore/exploit task. While males and females had similar performance, they used different latent explore-exploit strategies to learn about the dynamic environment. Males explored more than females and were more likely to "get stuck" in an extended period of exploration before committing to a favored choice. In contrast, females were more likely to show elevated learning during the exploratory period, making exploration more efficient and allowing them to start exploiting a favored choice earlier. Furthermore, the learning rate increased over days in females but stayed stable in males. Such meta-learning in females permitted learning about the current task (which option provides the best reward outcome), as well as the structure of the task (the reward probability of choices changes over time). This allowed them to shift more quickly to exploit a rewarding option when they found one and only explored when the current option failed to provide valuable rewards. 
369

370

371

372

373

374

375

376

377

378

379

380

Together, these results demonstrate that while the overall performance was similar, males and females tended to adopt different strategies for interacting with the same uncertain environment. The difference in explore-exploit strategies across sexes may provide us insight into potential sex-modulated mechanisms that are implicated in learning under uncertainty.

Our major finding that males learned less during exploration and explored for longer is consistent with two explanations. First, males learned more slowly during exploration and as a result, they had to explore for longer to learn which option was worth exploiting. Another possibility is that males got "stuck" in extended periods of exploration that prohibited them from applying the knowledge they have learned and committing to a rewarding option. In this view, it takes some energy to stop exploring and transition to exploit. As we have shown in our result, males had "deeper" exploratory states and they were more likely to keep exploring once started. These two explanations are not mutually exclusive because changes in learning could contribute to changes in the stickiness of exploration and vice versa. This essentially presents a chicken and egg problem. It is difficult to distinguish from behavior alone whether slower learning drives longer exploration, or vice versa, if being stuck in exploration results in slower learning. Neural measures during explore and exploit choices across sexes may help us differentiate learning signals from signals that drive exploration, and whether these signals are sex-different.

Answering these neural questions will require a way to reliably identify latent exploration and exploitation states. The Hidden Markov model (HMM) has been used to infer trial-by-trial exploration and exploitation in non-human primates (Ebitz et al., 2018). The HMM inferred latent goal states explained more variance in neural activity than other decision-variables (Ebitz et al., 2018). In our data, we have shown for the first time that the HMM model was able to label a meaningful exploratory state that matches normative definitions of exploration in the mouse model. In the future, this computational tool can complement neurobiological recording techniques to examine for neural correlates of exploration on a trial-by-trial basis, and permit the visualization of dynamic landscapes of choice behavior across individuals (as in Figure 2C) or with pharmacological or other challenges (Ebitz et al., 2019).

Reinforcement learning (RL) models have also been used in the past to identify levels of exploration across individuals (Daw et al., 2006; Pearson et al., 2009). However, our findings indicate that multiple latent parameters can influence how much these models explore. Here, we 
found that differences in exploration between the sexes were due to differences in learning rates, not due to differences in the decision noise parameter, which is more commonly associated with exploration. While RL models are helpful for understanding cognitive or computational mechanisms, they are limited in their ability to identify when exploration is happening. The HMM model, conversely, provides no insight into mechanisms, but can tell us precisely when exploration is occurring, both in animal behaviors and RL models (Ebitz et al., 2018). By combining the HMM and RL approaches, we capitalized on the advantages of both frameworks: linking changes in exploration across sexes to underlying mechanisms of exploration. Since the broader reinforcement learning model framework is highly adaptive and amenable, in the future, the HMM and kinds of model-free analyses we completed here could also inform the design of RL models to more precisely capture explore-exploit decisions.

Future work is needed to understand the neurobiological bases of these observations. One neuromodulator that is implicated in reinforcement learning, including the transition between exploration and exploitation, and is strongly sex-modulated is dopamine (Beeler et al., 2010; Jenni et al., 2017). Studies have shown that dopamine signals regulate exploration via mechanisms of action selection and learning (Beeler et al., 2010; Frank et al., 2009; Humphries et al., 2012). However, due to the exclusive use of males in many foundational experiments (Beeler et al., 2010; Cinotti et al., 2019), the fact that dopamine function on decision making is strongly modulated by sex and sex-linked mechanisms is often overlooked. For example, estradiol has been demonstrated to exert both acute and chronic modulatory effects on dopamine release, reuptake, and receptor binding (Yoest et al., 2014), allowing enhanced DA release and faster DA clearance in females (Becker, 1999). This mechanism could contribute to increased reward learning observed in females during exploration. The prefrontal cortex (PFC), which receives dopamine projections, is a target brain region to understand exploration and exploitation (Ebitz et al., 2018; Jenni et al., 2017). Our previous study implicated PFC in the differences in learning strategy between males and females (Chen et al., 2020). It is possible that prefrontal cortical dopamine is particularly engaged in implementing explore-exploit strategies via sexbiased mechanisms of learning.

A frequent use of rodent operant testing is to perform cognitive assessments with similar rule structures in animals and dependent measures as are used in human tasks, to permit 
429 translational work in animals exploring pharmacology, genetics, and other potential biological

430 contributors to behavior (Grissom and Reyes, 2018; Heath et al., 2016). However, the vast

431 majority of these tasks were not designed with computational models in mind, and as a result, we

432 are unable to assess whether similar latent cognitive variables are influencing behavior in

433 humans and rodents. Here, we establish that explore-exploit decisions are shared cognitive

434 processes across species, making this restless bandit task an ideal tool for translational research.

435 Parallel approaches in humans have been used to examine the explore-exploit strategic

436 phenotype of neuropsychiatric disorders, including ADHD, addiction, and depression.(Addicott

437 et al., 2020, 2012; Beeler et al., 2012; Blanco et al., 2013). The computational modeling used

438 here permits fine grained quantification of individual variability in latent parameters that capture

439 adaptive changes in exploration in changing environments. The computational approaches we

440 develop here could help identify behavioral endophenotypes across species underlying a variety

441 of neuropsychiatric disorders, and open up new avenues for understanding as well as rescuing

442 dysfunction in value-based decision making. 


\section{Figure 1}

445

A
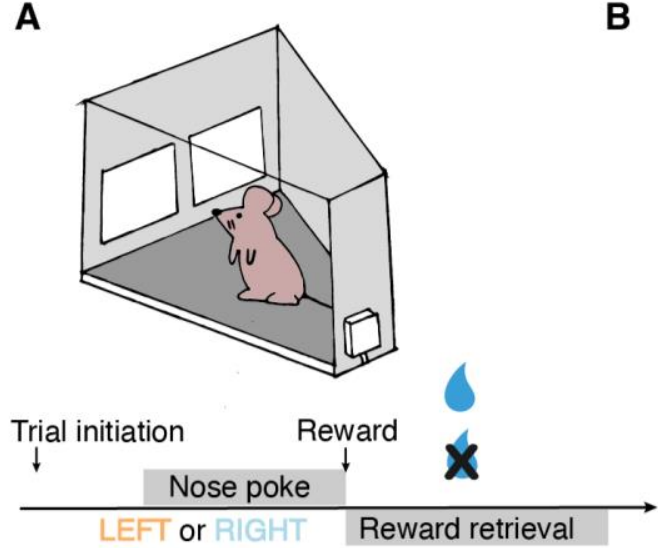

D

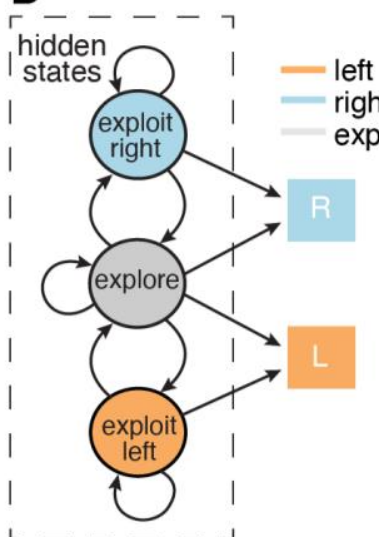

ட

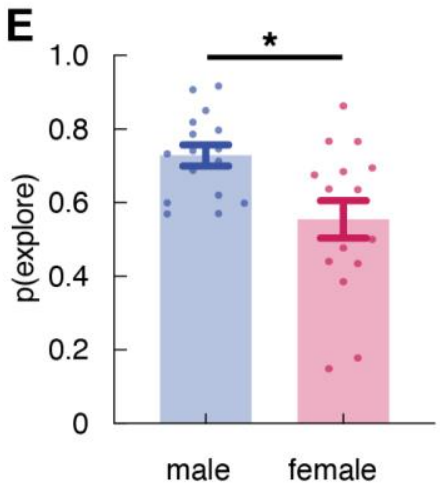

B

$\mathbf{F}$
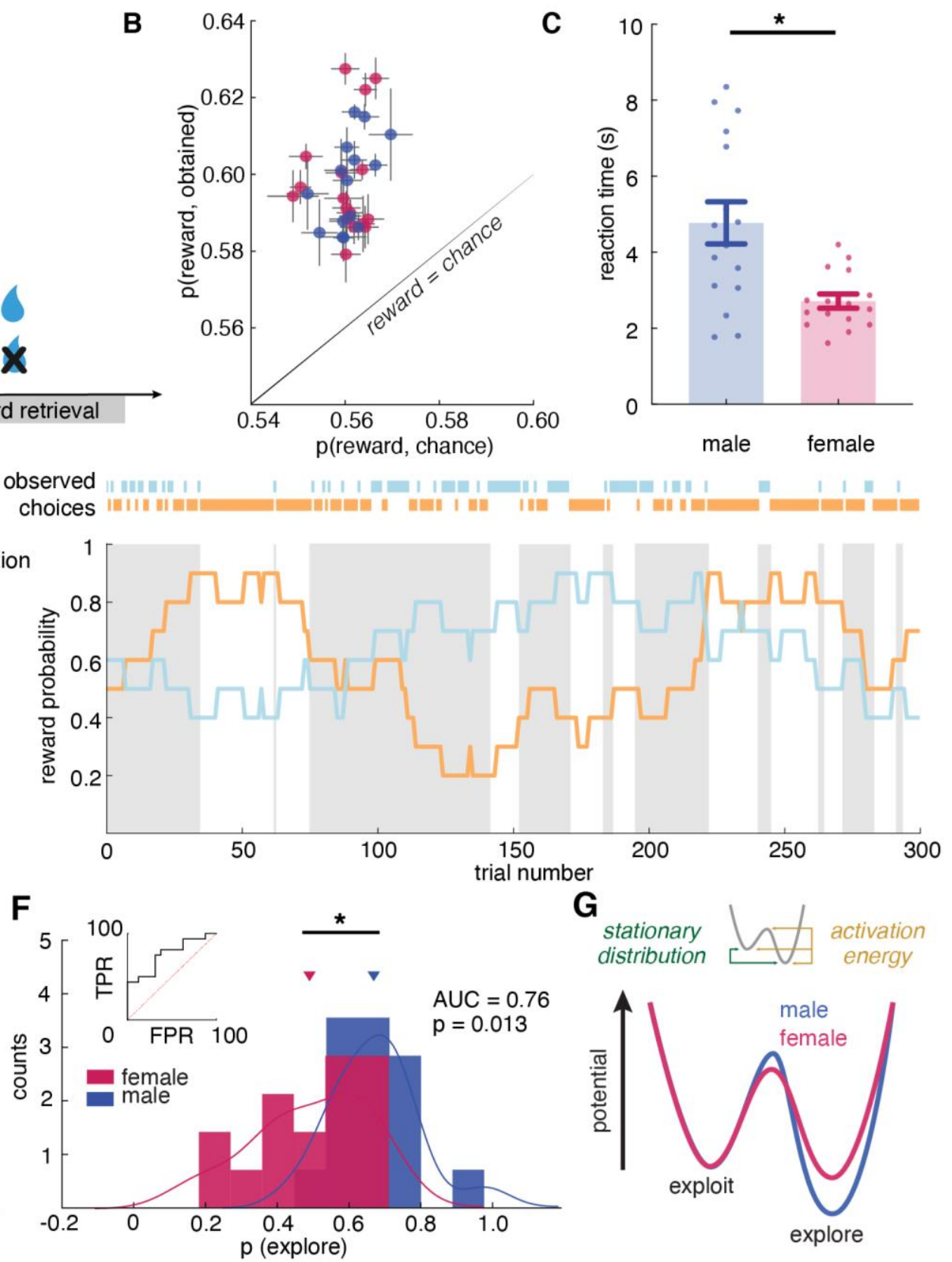

G stationary distribution
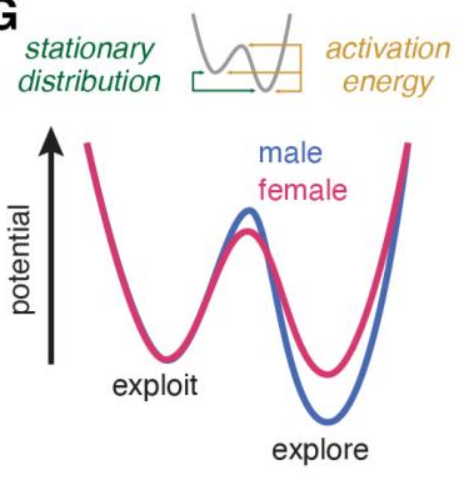

Figure 1. Male and female mice showed different exploratory strategies in a restless bandit started.

A) Schematic of the mouse touchscreen chamber with the restless two-armed bandit task and trial structure. B) Average probability of obtaining reward compared to the chance 
452 probability of reward across individuals (dots). C) Average reaction time of both sexes. Females 453 responded significantly faster than did males. D) (left) A hidden Markov model that labeled 454 exploration and exploitation as latent goal states underlying observed choices. This model 455 includes an exploitation state for each arm and an exploration state where the subject chooses 456 one of the arms randomly. (right) Reward probabilities (lines) and choices (dots) for 300 457 example trials for a given mouse. Shaded areas highlight explore-labeled choices. E, F) Average 458 (E) and distribution (F) of percentage of Hidden Markov Model (HMM)-labeled exploratory 459 trials in females and males. G) Dynamic landscape of the fitted HMMs for males and females. 460 The model fit to males had deeper exploratory states, with higher activation energy between the 461 states. * indicates $\mathrm{p}<0.05$. Graphs depict mean \pm SEM across animals. 
Figure 2

A

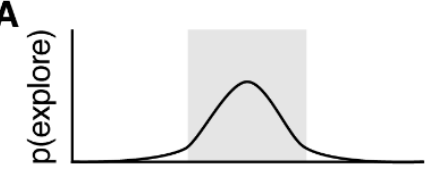

value 1 - value 2

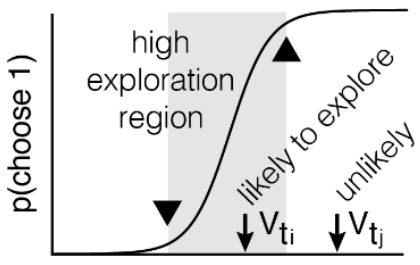

value 1 - value 2

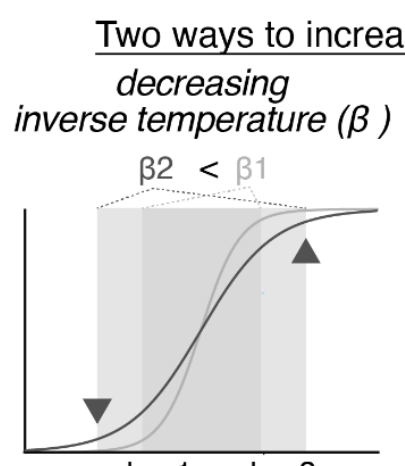

value 1 - value 2

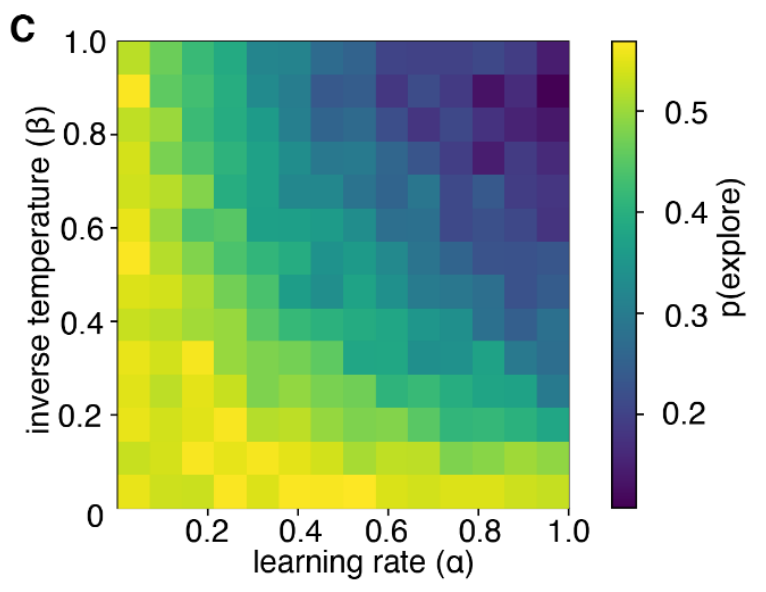

463

B
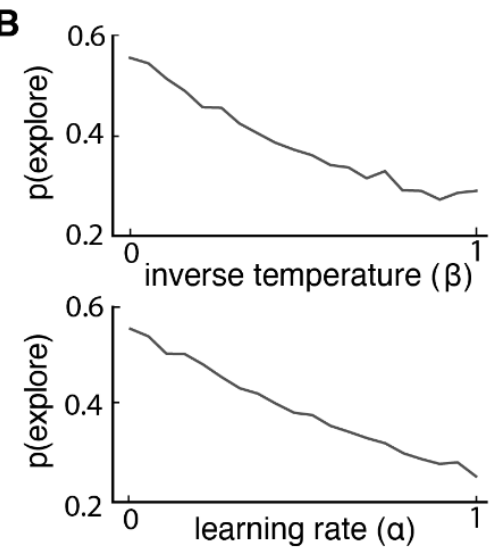

Figure 2. Multiple reinforcement learning parameters can influence the probability of exploration.

A) Exploration occurs most often when option values are close together, illustrated by the gray shaded boxes in the value-choice functions. Both decreasing inverse temperature $(\beta)$ and decreasing learning rate increases exploration because each manipulation changes the amount of time spent in the high exploration zone, although the mechanisms are different. Decreasing inverse temperature $(\beta)$ widens the zone by flattening the value-choice function and increasing

471 decision noise. Decreasing learning rates $(\alpha)$ keeps learners in the zone for longer. B) Probability

472 of exploration from 10,000 different reinforcement learning agents performing this task,

473 initialized at different random combinations of inverse temperatures $(\beta)$ and learning rates $(\alpha)$.

474 Marginal relationships between decision noise (top) and learning rate (bottom) are shown here.

475 C) Heatmap of all pairwise combinations of learning rate and inverse temperature. 
A

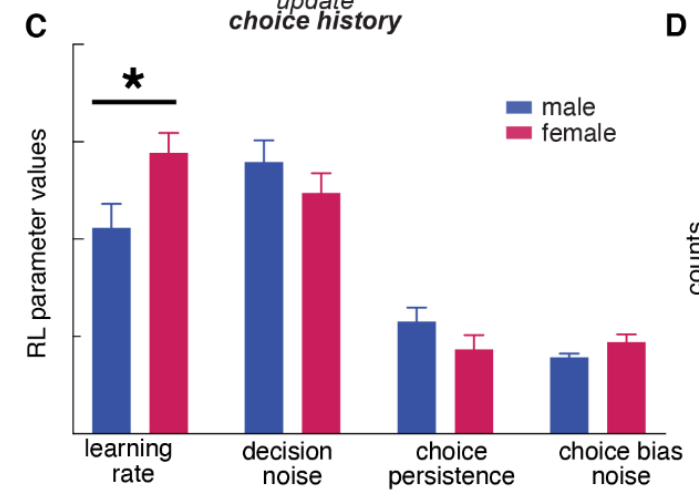

B

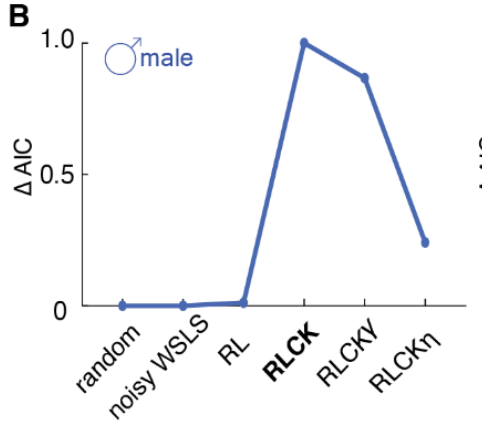

D

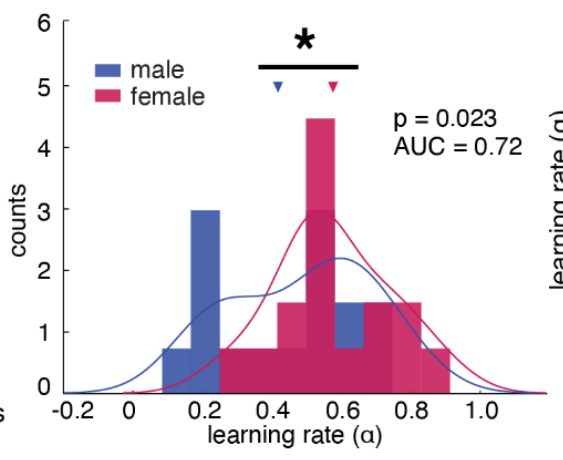

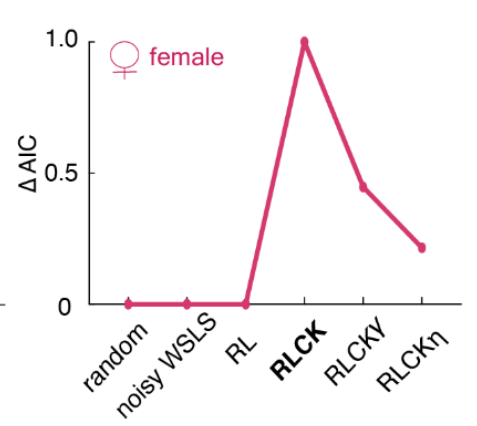

E

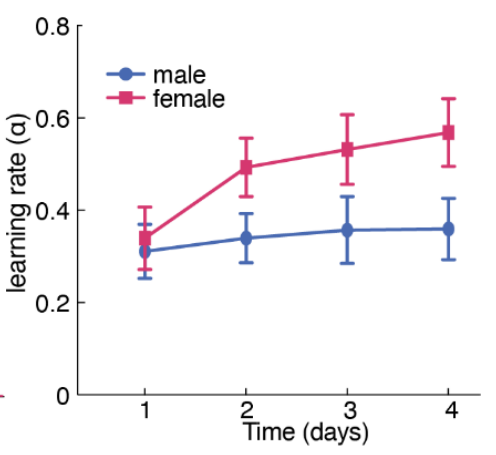

Figure 3. Sex differences in learning rate, but not decision noise, drove differences in explore-exploit decisions.

A) A diagram of latent parameters that capture learning $(\alpha)$, bias $\left(\alpha_{c}\right)$, inverse temperature $(\beta)$ in reinforcement learning models. The models tested used a combination of these parameters (see Methods). B) Model comparison across 6 reinforcement learning models with various parameter combinations. The four-parameter reinforcement learning-choice kernel (RLCK) model has the highest relative likelihood in both males and females. C) All four parameters in the best fit RLCK model across sexes. Learning rate $(\alpha)$ was significantly higher in females than males. D) Distribution of learning rate across sexes. E) Learning rate in females increased across

491 animals. 
493 Figure 4
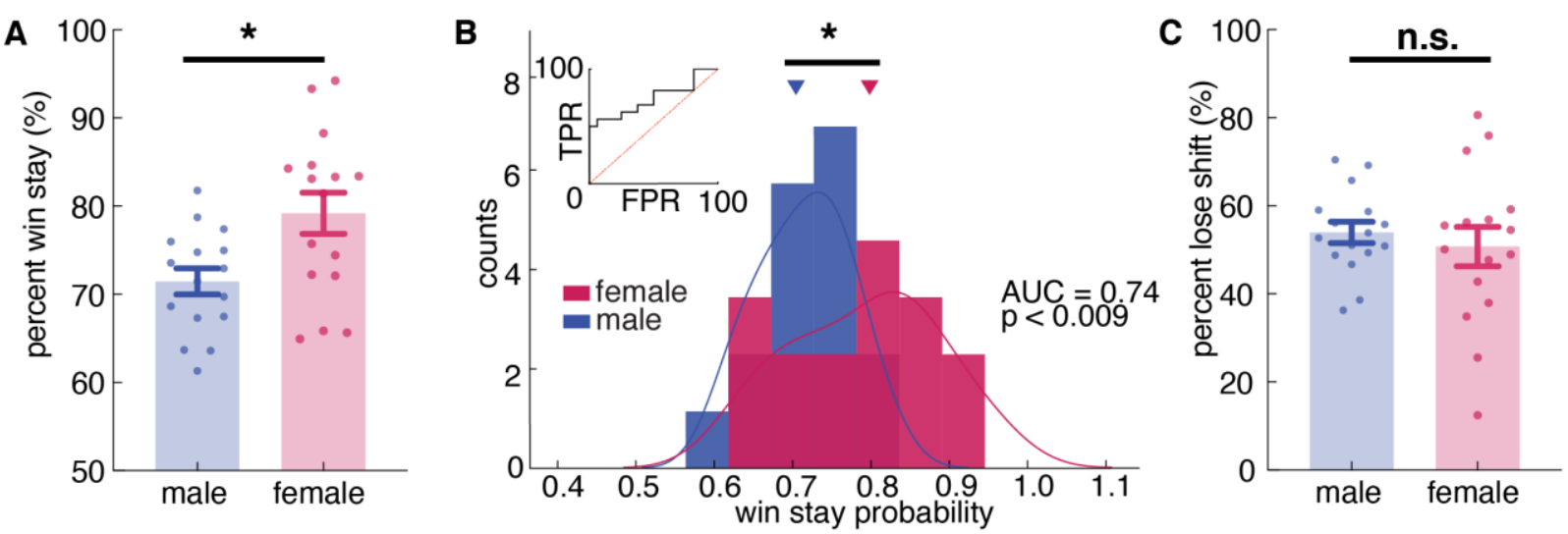

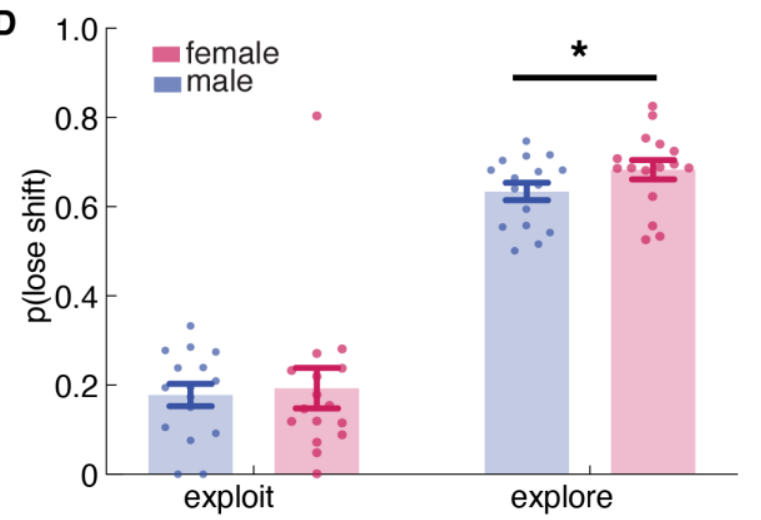

$\mathbf{F}$

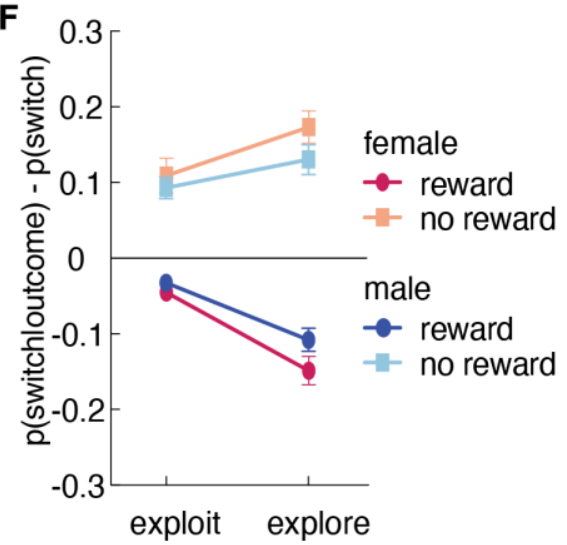

G
E
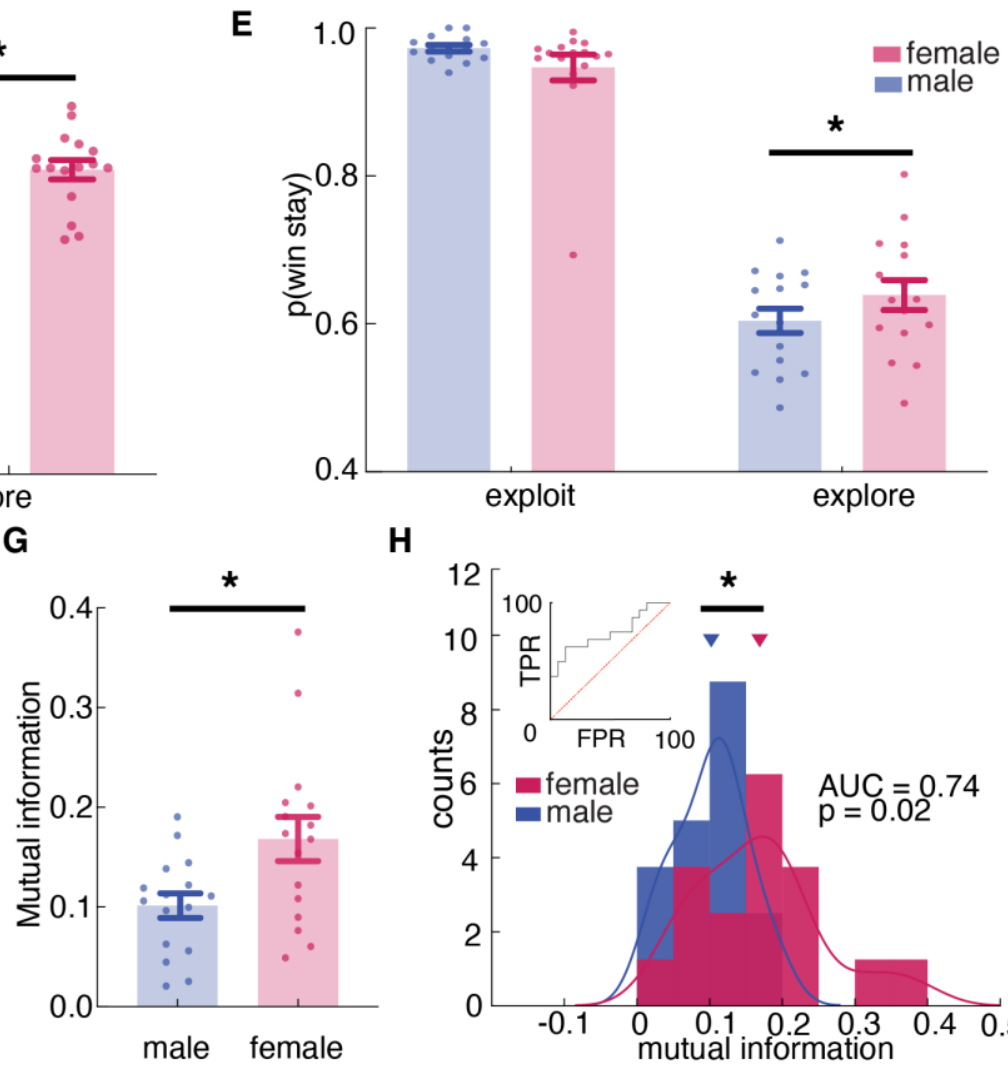

H

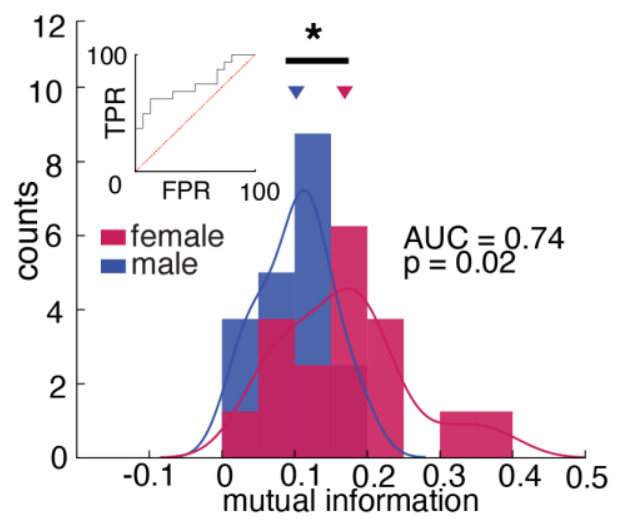

Figure 4. Females used more information about past outcomes and past choices to make decisions, and learned more during exploration.

A, B) Percent win stay behavior (A: average; B: distribution) reveals that females were more likely to stay with the same choice after a reward. C) Average percentage of lose shift behavior across sexes. D) Probability of shifting after a loss during an explore or exploit trials. E) Probability of staying after a win during an explore or exploit trials. F) during explore and exploit trials. The probability of males and females switching targets on the next trial, given the 
502 current trial's outcome and latent state. Females only learned more during exploratory trials. G, 503 H) Average $(\mathrm{G})$ and distribution $(\mathrm{H})$ of percentage of mutual information across all trials in 504 females and males reveals that females use more information about past trials (choice and 505 outcome) in making future decisions. * indicates $\mathrm{p}<0.05$. Graphs depict mean \pm SEM across 506 animals. 


\section{Supplemental Figures}
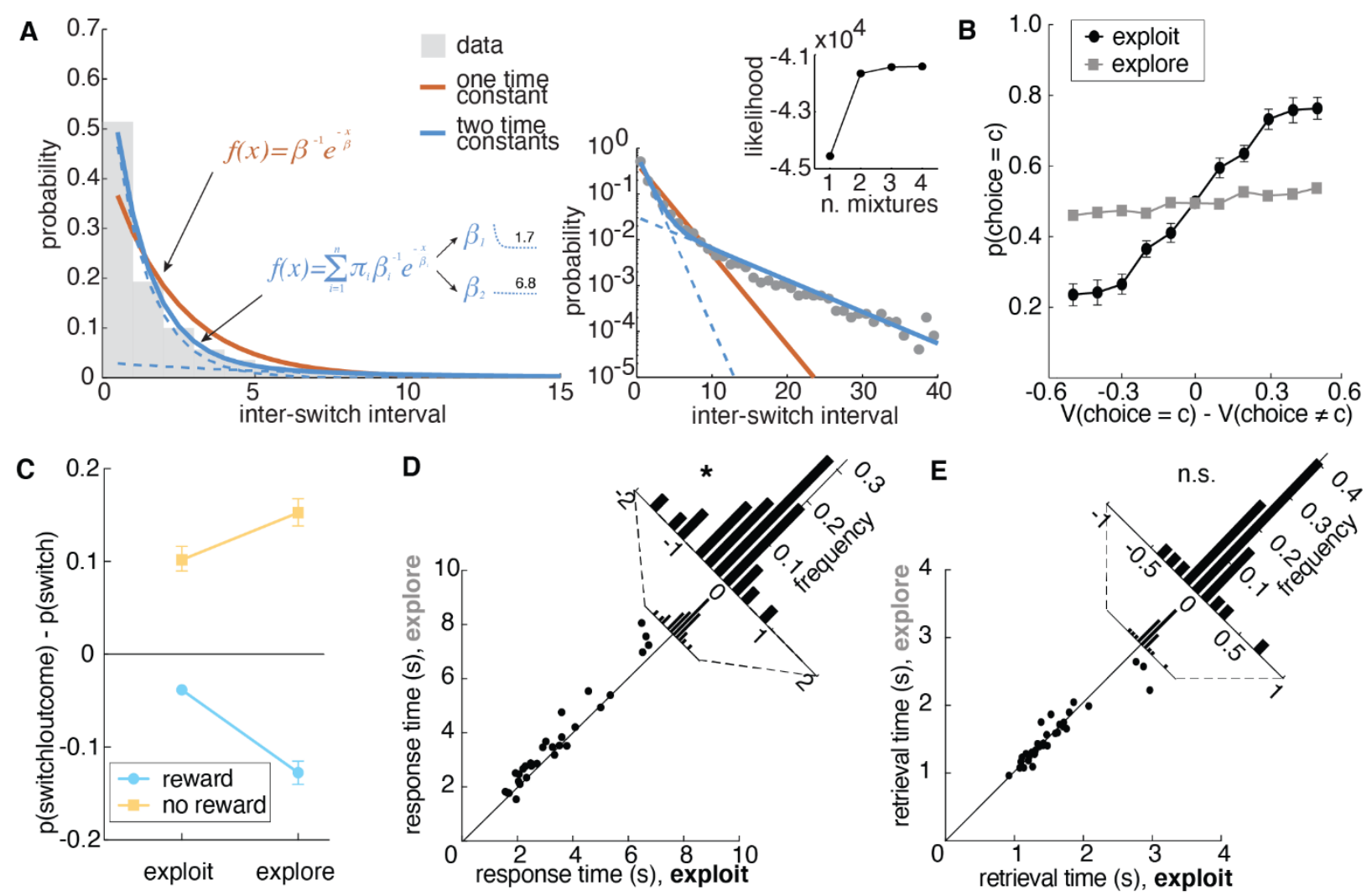

Supplemental Figure 1. Two time constants combined best describe the rate of switching choices in animals' choice behavior and Hidden Markov model validation. Related to Figure 1D.

A) The distribution of times between switch decisions (inter-switch intervals). A single probability of switching would produce exponentially distributed inter-switch intervals. Orange

514 line, the maximum likelihood fit for a single discrete exponential distribution. Solid blue line, a

515 mixture of two exponential distributions, with each component distribution in dotted blue. The

516 two components reflect one fast-switching time constant (average interval, 1.7 trials) and one

517 persistent time constant ( 6.8 trials). The right plot is the same as the left, but with a log scale.

518 Inset is the log likelihood of mixtures of different numbers of exponential distributions. B)

519 Probability of choice as a function of value differences between choices for exploratory and 520 exploitative states. C) The probability of animals switching targets on the next trial, given the 521 current trial's outcome and latent state. D) Difference in choice response time between explore 522 and exploit choices. E) Difference in choice response time between explore and exploit choices.

523 There is no significant difference in retrieval time between two latent states, suggesting that 
bioRxiv preprint doi: https://doi.org/10.1101/2020.12.29.424773; this version posted April 23, 2021. The copyright holder for this preprint (which was not certified by peer review) is the author/funder. All rights reserved. No reuse allowed without permission.

524 exploration was not merely disengagement from the task. * indicates $\mathrm{p}<0.05$. Graphs depict 525 mean \pm SEM across animals. 
A Animal or model percent choice compared to the matching law
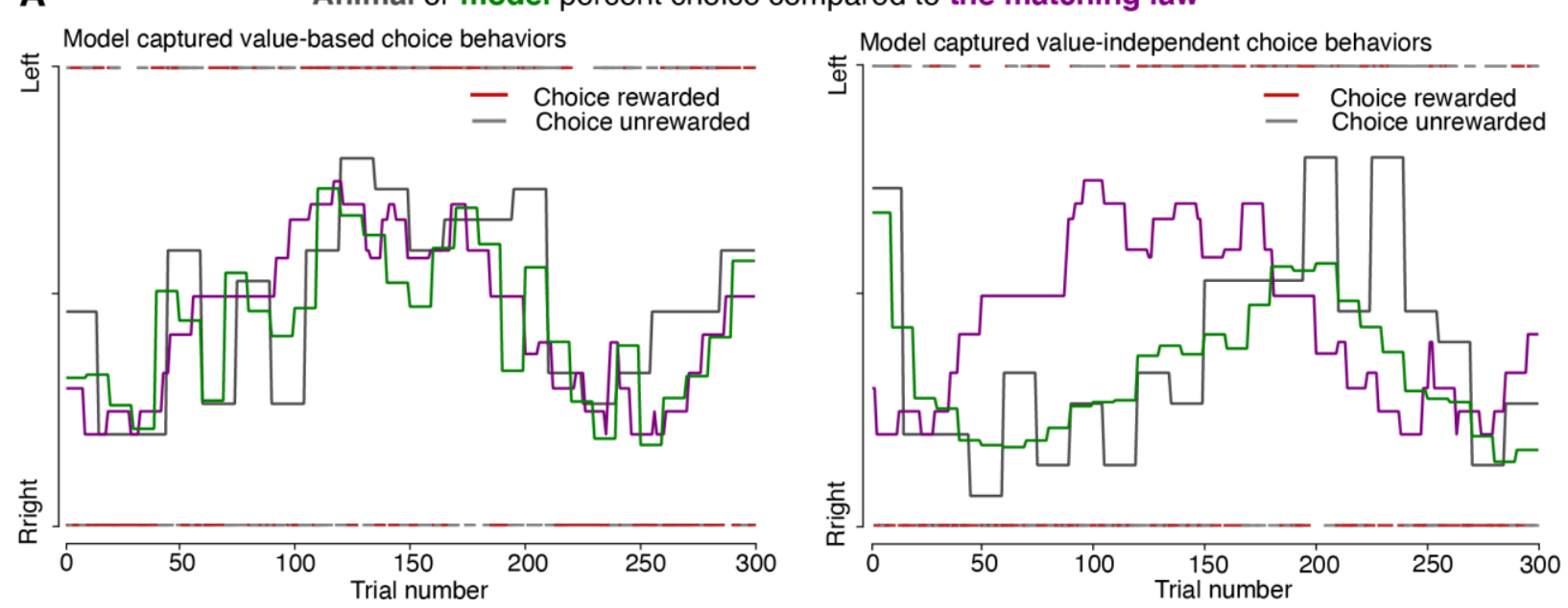

528 Supplemental Figure 2. The best fit model, the four-parameter reinforcement learning-

529 choice kernel (RLCK) model, captured both value-dependent and value-independent

530 choice behaviors. Actual choices (gray) and simulated choices (green) from the best fit model

531 (RLCK) of two example animals. Predictions from the matching law are illustrated as a contrast

532 to the best-fitting RL model. 


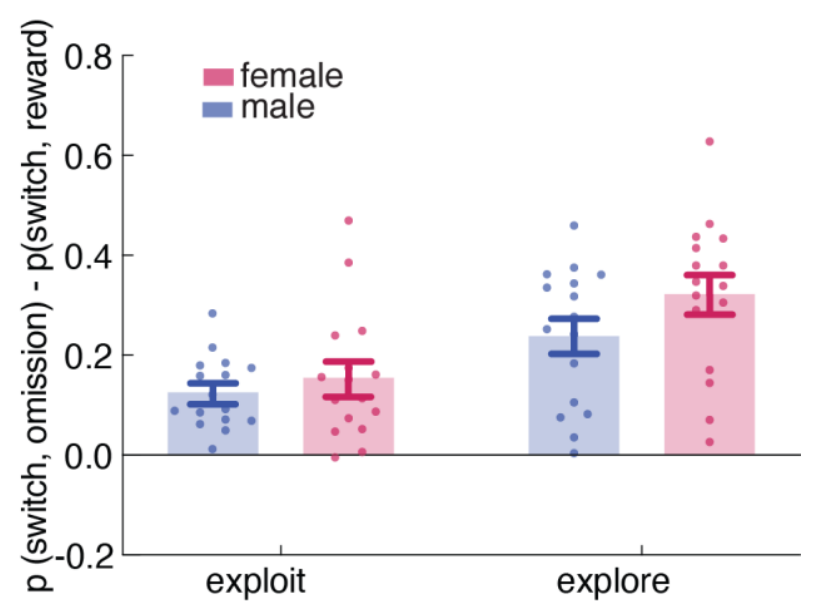

534 Supplemental Figure 3. Reward learning in explore vs. exploit state across sexes. Related to

535 Figure 4F. The probability of males and females switching targets on the next trial, given the

536 current trial's outcome and latent state. Females showed increased reward learning only during 537 exploratory state. 
538 Table 1: Main effects and interaction effects (pairwise and 3-way) of sex, outcome, latent 539 state, and subject identity.

Table 1: Four-way Repeated Measures ANOVA

\begin{tabular}{l|ccccc}
\hline Effect & Sum Sq. & df. & Mean Sq. & F & p value \\
\hline sex & 0.0782 & 1 & 0.0782 & 6.89 & 0.0088 \\
subject(sex) & 2.1709 & 30 & 0.0904 & 7.95 & $<0.00001$ \\
reward & 13.1509 & 1 & 13.1509 & 1157.63 & $<0.00001$ \\
state & 35.4862 & 1 & 35.4862 & 3123.74 & $<0.00001$ \\
sex x reward & 0.2094 & 1 & 0.2094 & 18.44 & $<0.00001$ \\
sex x state & 0.0383 & 1 & 0.0383 & 3.37 & 0.0667 \\
subject(sex) x reward & 3.616 & 30 & 0.1205 & 10.61 & $<0.00001$ \\
subject(sex) x state & 1.8529 & 30 & 0.0618 & 5.44 & $<0.00001$ \\
reward x state & 0.4652 & 1 & 0.4652 & 40.95 & $<0.00001$ \\
sex x reward x state & 0.0463 & 1 & 0.0463 & 4.07 & 0.0438 \\
subject(sex) x reward x state & 0.5573 & 30 & 0.0186 & 1.64 & 0.0177 \\
\hline error & 9.872 & 869 & 0.0114 & & \\
\hline total & 68.5207 & 996 & & & \\
\hline
\end{tabular}




\section{Methods}

Animals. Thirty-two BL6129SF1/J mice (16 males and 16 females) were obtained from Jackson Laboratories (stock \#101043). Mice arrived at the lab at 7 weeks of age, and they were housed in groups of four with ad libitum access to water while being mildly food restricted (85$95 \%$ of free feeding weight) for the experiment. Animals engaging in operant testing were housed in a 0900-2100 hours reversed light cycle to permit testing during the dark period. Before operant chamber training, animals were food restricted to $85 \%-90 \%$ of free feeding body weight. Operant testing occurred five days per week (Monday-Friday). All animals were cared for according to the guidelines of the National Institution of Health and the University of Minnesota.

Apparatus. Sixteen identical triangular touchscreen operant chambers (Lafayette Instrument Co., Lafayette, IN) were used for training and testing. Two walls were black acrylic plastic. The third wall housed the touchscreen and was positioned directly opposite the magazine. The magazine provided liquid reinforcer (Ensure) delivered by a peristaltic pump, typically $7 \mathrm{ul}$ (280 ms pump duration). ABET-II software (Lafayette Instrument Co., Lafayette, IN) was used to program operant schedules and to analyze all data from training and testing.

Behavioral task. Two-armed spatial restless bandit task. Animals were trained to perform a two-armed spatial restless bandit task in the touchscreen operant chamber. Each trial, animals were presented with two identical squares on the left and right side of the screen. Nose poke to one of the target locations on the touchscreen was required to register a response. Each location is associated with some probability of reward, which changes independently over time. For every trial, there is a $10 \%$ chance that the reward probability of a given arm will increase or decrease by $10 \%$. All the walks were generated randomly with a few criteria: 1) the overall reward probabilities of two arms are within $2 \%$ of each other, preventing one arm being overly better than the other, 2) the reward probability cannot go down to $0 \%$ or go up to $100 \%, 3$ ) there are no 30 consecutive trials where the reward probabilities of both arms are lower than $20 \%$ to ensure motivation. Animals ran a simple deterministic schedule on Monday to re-adapt to operant chamber after weekends off and ran a different restless bandit task each day from Tuesday to Friday. Animals ran for 2 rounds of 4 consecutive days and within each day, animals completed 
572 either 300 trials or spent a maximum of two hours in the operant chamber. Data was recorded by

573 the ABET II system and was exported for further analysis. All computational modeling was

574 conducted using python.

\section{Data analysis}

General analysis techniques. Data was analyzed with custom PYTHON, MATLAB, and

Prism 8 scripts. Generalized linear models, ANOVA, and t-test were used to determine sex differences over time, unless otherwise specified. P values were compared against the standard a $=0.05$ threshold. The sample size is $\mathrm{n}=16$ for both males and females for all statistical tests. No animal was excluded from the experiment. One outlier was removed in one analysis using ROUT method (with Q set to 1\%). This outlier was from the animal that ran the lowest number of total trials. Statistics for both no outlier removal and outlier removal were reported in the result. All statistical tests used and statistical details were reported in the results. All figures depict mean \pm SEM.

Mixture model. We first asked whether there were different behavioral dynamics that might correspond to exploration and exploitation. Exploration and exploitation take place on different time scales. In RL agents, for example, exploration is typically implemented via adding noise or indeterminacy to a decision-rule. The identity of choices that are caused by this noisethe exploratory choices - will thus switch more frequently than the identity of choices that

592 depend on option value. We should see short runs of exploratory choices and long runs of exploitative ones (Ebitz et al., 2018). To the extent that choice runs end probabilistically (an assumption of the HMM framework), choice run durations (inter-switch intervals) will be exponentially distributed (Berg, 1993). To the extent that there are multiple causal regimes (such as exploration and exploitation), inter-switch intervals will be distributed as a mixture of multiple

597 exponential distributions (Supplemental Figure 1). Because trials are discrete, rather than

598 continuous, we fit mixtures of the discrete equivalent to the exponential distribution, the geometric distribution. We examined the distribution of 24,836 interswitch intervals. Adding a

600 second mixing distribution significantly improved model fit (1-component, 1-parameter mixture 601 log-likelihood: -44555, 2-component, 3-parameter: -41656; likelihood ratio test, $\mathrm{p}<10^{-32}$ ).

602 Adding additional mixing distributions continued to improve model fit, a common observation in 
603 mixture modeling. However, the continued improvement was substantially less than the leap

604 from 1 to 2 components (Supplemental Figure 1; 3-component: -41431, 4-component: -41412)

605 and additional mixtures beyond 2 had weights below 3\%. This suggests that a mixture of one

606 fast-switching regime and one slow-switching regime was the most parsimonious explanation for 607 the data (McLachlan and Peel, 2004).

Hidden Markov model (HMM). In order to identify when animals were exploring or 610 exploiting, we fit an HMM. This differs from the approach used in some previous studies, where 611 reinforcement learning (RL) models were used to quantify exploration (Cinotti et al., 2019; Daw 612 et al., 2006; Ishii et al., 2002; Jepma and Nieuwenhuis, 2011; Pearson et al., 2009). These studies 613 typically took one of two approaches. In the first, individual variability in exploration is inferred 614 from variability in a specific RL model parameter thought to be linked to exploration (i.e. inverse 615 temperature/decision-noise (Cinotti et al., 2019; Ishii et al., 2002; Schweighofer and Doya, 616 2003). However, changing decision-noise is not the only way to change how often an RL agent

617 explores (Figure 2), meaning that the inverse temperature/decision-noise parameter does not 618 uniquely measure exploration. In the second approach, an RL model is fit to behavior and 619 decisions that deviate from model values are labeled as exploratory (Daw et al., 2006; Jepma and 620 Nieuwenhuis, 2011; Pearson et al., 2009). This approach is based on the rationale that 621 exploration is a non-reward maximizing goal. However, a non-reward maximizing goal would 622 produce choices that are orthogonal to reward, not the errors of reward maximization identified 623 by this method (Averbeck et al., 2017; Ebitz et al., 2018). Beyond issues of face validity, both 624 approaches are sensitive to any misspecifications in the behavioral model: a subject who deviates 625 more from the assumptions of the model may seem to explore more since the subject will also 626 have high decision-noise and a large number of choices will not match the model's predictions.

627 For these reasons, we chose to apply an HMM approach, which models exploration directly, 628 rather than inferring it from collateral observations, and does not depend on any kind of 629 generative model of how the animals perform the task--a salient concern here, where we suspect 630 that these computations differ between the sexes. 
634 transitioning from each state to every other state. Our model consisted of two types of states, the 635 explore states and the exploit state. The emissions model for the explore state was uniform across 636 the options:

$$
p\left(y_{t}=k \mid z_{t}=\text { explore }\right)=\frac{1}{N_{k}}
$$

This is simply the maximum entropy distribution for a categorical variable and does not imply or enforce that exploratory choices were random choices (Ebitz et al., 2020, 2019).

640 Because exploitation involves repeated samples to each option, exploit states only permitted 641 choice emissions that matched one option. That is: transition matrix. This matrix is a system of stochastic equations describing the 1-time-step probability of transitioning between every combination of past and future states $(i, j)$. were tied across exploit states such that each exploit state had the same probability of beginning (from exploring) and of sustaining itself. Transitions out of the exploration, into exploitative states, were similarly tied. The model also assumed that the mice had to pass through exploration 654 in order to start exploiting a new option, even if only for a single trial. Through fixing the emissions model, constraining the structure of the transmission matrix, and tying the parameters,

656 the final HMM had only two free parameters: one corresponding to the probability of exploring, 657 given exploration on the last trial, and one corresponding to the probability of exploiting, given exploitation on the last trial. 
662 but also initial distribution over states, typically denoted as PI. Because the mice had no

663 knowledge of the environment at the first trial of the session, we assumed they began by

664 exploring, rather than add another parameter to the model here. The algorithm was reinitialized

665 with random seeds 20 times, and the model that maximized the observed (incomplete) data log

666 likelihood across all the sessions for each animal was ultimately taken as the best. To decode

667 latent states from choices, we used the Viterbi algorithm to discover the most probable a

668 posteriori sequence of latent states.

Analyzing model dynamics. In order to understand how exploration and exploitation changed across males and females, we analyzed the HMMs. The term "dynamics" means the rules or laws that govern how a system evolves over time. Here, the system of interest was

672 decision making behavior, specifically at the level of the hidden explore and exploit goals. In

673 fitting our HMMs, we were fitting a set of equations that describe the dynamics of these goals:

674 the probability of transitions between exploration and exploitation and vice versa. Of course,

675 having a set of fitted equations is a far cry from understanding them. To develop an intuition for

676 how sex altered the dynamics of exploration, we therefore turned to analytical tools that allowed

677 us to directly characterize the energetic landscape of behavior (Figure 2C).

In statistical mechanics, processes within a system (like a decision-maker at some moment in time) occupy states (like exploration or exploitation). States have energy associated

680 with them, related to the long-time scale probability of observing a process in those states. A low 681 energy state is one that is very stable and deep, much like a valley between two high peaks. Low 682 energy states will be over-represented in the system. A high energy state, like a ledge on the side 683 of a mountain, is considerably less stable. High energy states will be under-represented in the 684 long-term behavior of the system. The probability of observing a process in a given state $\mathrm{i}$ will is 685 related to the energy of that state $\left(\mathrm{E}_{\mathrm{i}}\right)$ via the Boltzman distribution:

$$
p_{i}=\frac{1}{Z} e^{\frac{-E_{i}}{k_{B} T}}
$$

Where $\mathrm{Z}$ is the partition function of the system, $\mathrm{k}_{\mathrm{B}}$ is the Boltzman constant, and $\mathrm{T}$ is the 688 temperature of the system. If we focus on the ratio between two state probabilities, the partition 689 functions cancel out and the relative occupancy of the two states is now a simple function of the 690 difference in energy between them: 


$$
\frac{p_{i}}{p_{j}}=e^{\frac{-\left(E_{i}-E_{j}\right)}{k_{B} t}}
$$

Rearranging, we can now express the difference in energy between two states as a function of the difference in the long-term probability of those states being occupied:

$$
\ln \left(\frac{p_{i}}{p_{j}}\right) k_{B} T=E_{j}-E_{i}
$$

Meaning that the difference in the energetic depth of the states is proportional to the natural $\log$ of the probability of each state, up to some multiplicative factor $k_{B} T$. To calculate the probability of exploration and exploitation $\left(\mathrm{p}_{\mathrm{i}}\right.$ and $\left.\mathrm{p}_{\mathrm{j}}\right)$, we calculated the stationary distribution of the fitted HMMs. The stationary distribution is the equilibrium probability distribution over states. This means that this distribution is the relative frequency of each state that we would observe if the model's dynamics were run for an infinite period of time. Each entry of the model's transition matrix reflects the probability that the mice would move from one state (e.g. exploring) to another (e.g. exploiting one of the options) at each moment in time. Because the parameters for all the exploitation states were tied, each transition matrix effectively had two states - an explore state and a generic exploit that described the dynamics of all exploit states. Each of the $k$ animals had its own transition matrix $\left(A_{k}\right)$, which describes how the entire system - an entire probability distribution over states - would evolve from time point to time point. We observe how the dynamics evolve any probability distribution over states $(\pi)$ by applying the dynamics to this distribution:

$$
\pi_{t+1}=\pi_{t} A_{k}
$$

711 unchanged by continued application of the transition matrix as the distribution of states reaches

712 its equilibrium. That is, in stationary systems, there exists a stationary distribution, $\pi^{*}$, such that:

$$
\pi^{*}=\pi^{*} A_{k}
$$

If it exists, this distribution is a (normalized) left eigenvector of the transition matrix $A_{k}$

715 with an eigenvalue of 1 , so we solved for this eigenvector to determine the stationary distribution

716 of each $A_{k}$. Whe then took an average of these stationary distributions within each sex, plugged

717 them back into the Boltzman equations to calculate the relative energy (depth) of exploration and 718 exploitation as illustrated in Figure 2C. 
In order to understand the dynamics of our coarse-grained system, we need to not only understand the depth of the two states, but also the height of the energetic barrier between them: the activation energy required to transition from exploration to exploitation and back again.

722 Here, we build on an approach from chemical kinetics that relates the rate of transition between 723 different conformational states to the energy required to affect these transitions. The Arrhenius 724 equation relates the rate of transitions away from a state (k) to the activation energy required to 725 escape that state $\left(E_{a}\right)$ :

$$
k=A e^{\frac{E_{a}}{k_{B} T}}
$$

Where $\mathrm{A}$ is a constant pre-exponential factor related to the readiness of reactants to undergo the transformation. We will set this to 1 for convenience. Again, $\mathrm{k}_{\mathrm{B}} \mathrm{T}$ is the product of temperature and the Boltzman constant. Note the similarities between this equation and the Boltzman distribution illustrated earlier. Rearranging this equation to solve for activation energy 731 yields:

$$
E_{a}=-\ln \left(\frac{k}{A}\right) k_{B} T
$$

Thus, much like the relative depth of each state, activation energy is also proportional to some measurable function of behavior, up to some multiplicative factor $\mathrm{k}_{\mathrm{B}} \mathrm{T}$. Note that our approach has only identified the energy of three discrete states (an explore state, an exploit state, and the peak of the barrier between them). These are illustrated by tracing a continuous potential through these three points only to provide a physical intuition for these effects. could potentially characterize animals' choice behaviors, with details of each RL model as

741 below. To identify the model that best captured the computations used by the animals, we 742 compared model fits across six reinforcement learning models with different combinations of

743 latent parameters. AIC weights were calculated from AIC values of each model for each sex and 744 compared across models to determine the best model with the highest relative likelihood.

745 The first model assumes that animals choose between two arms randomly with some 746 overall bias for one side over the other. This choice bias for choosing left side over right side is 747 captured with a parameter $b$. The probability of choosing left side on trial $t$ is: 
The second model is a noisy win-stay lose-shift (WSLS) model that adapts choices with regards to outcomes. This model assumes a win-stay lose-shift policy that is to repeat a rewarded choice and to switch to the other choice if not rewarded. Furthermore, this model includes a parameter $\epsilon$ that captures the level of randomness, allowing a stochastic application of the win-stay lose-shift policy. The probability of choosing arm $k$ on trial $t$ is:

$p_{t}^{k}=\left\{\begin{array}{c}1-\frac{\epsilon}{2}, \text { if }\left(c_{t-1}=k \text { and } r_{t-1}=1 \text { OR } c_{t-1} \neq k \text { and } r_{t-1}=0\right) \\ \frac{\epsilon}{2}, \text { if }\left(c_{t-1} \neq k \text { and } r_{t-1}=1 \text { OR } c_{t-1}=k \text { and } r_{t-1}=0\right)\end{array}\right.$ [2] "noisy WSLS"

$c_{t}$ indicates the choice on trial $t$ and $r_{t}$ is a binary variable that indicates whether or not trial $t$ was rewarded. two-parameter model assumes that animals learn by consistently updating $Q$ values, which are values defined for options (left and right side). These $\mathrm{Q}$ values, in turn, dictate what choice to make next. For example, in a multi-armed bandit task, $Q_{t}{ }^{k}$ is the value estimation of how good $\operatorname{arm} k$ at trial $t$, and is updated based on the reward outcome of each trial:

$$
Q_{t+1}^{k}=Q_{t}^{k}+\alpha\left(r_{t}-Q_{t}^{k}\right)
$$

In each trial, $\mathrm{r}_{\mathrm{t}}-\mathrm{Q}_{\mathrm{t}}{ }^{\mathrm{k}}$ captures the reward prediction error (RPE), which is the diffe-rence between expected outcome and the actual outcome. The parameter $a$ is the learning rate, which determines the rate of updating RPE. With Q values defined for each arm, choice selection on each trial was performed based on a Softmax probability distribution:

$$
p\left(a_{t+1}=k\right)=\frac{e^{\beta Q_{t}^{k}}}{\sum_{j} e^{\beta Q_{t}^{j}}}
$$
rule in model 3. The model assumes that choice kernel, which captures the outcome-independent tendency to repeat a previous choice, also influences decision making. The choice kernel updating rule is similar to the value-updating rule: and $a_{\mathrm{t}}$ is choice kernel updating rate, characterizing choice persistence. The value and choice

$$
C K_{t+1}^{k}=C K_{t}^{k}+\alpha_{c}\left(a_{t}^{k}-C K_{t}^{k}\right)
$$$$
\text { [4] " } R L C K \text { ” }
$$

, where $\mathrm{a}_{\mathrm{t}}^{\mathrm{k}}$ is a binary variable that indicates whether or not arm $k$ was chosen on trial $t$ kernel term were combined to compute the probability of choosing arm $k$ on trial $t$ : 


$$
p_{t}^{k}=\frac{e^{\left(\beta Q_{t}^{k}+\beta_{c} C K_{t}^{k}\right)}}{\sum_{j} e^{\left(\beta Q_{t}^{j}+\beta_{c} C K_{t}^{j}\right)}}
$$

779 stickiness of choice.

The fifth model is the same with the fourth model, except that this model includes another parameter $\gamma$ that modulates learning rate when the choice is not rewarded. This model assumes that the learning rate is different for rewarded and unrewarded trials.

$$
Q_{t+1}^{k}=\left\{\begin{array}{c}
Q_{t}^{k}+\alpha\left(r_{t}-Q_{t}^{k}\right), r_{t}=1 \\
Q_{t}^{k}+\gamma \times \alpha\left(r_{t}-Q_{t}^{k}\right), r_{t}=0
\end{array}\right.
$$

The sixth model is also similar to model 4, except that this model includes another parameter $\eta$ that tunes the balance between the value updating rule and the choice kernel updating rule. This model assumes that animals could be using two policies (value and choice kernel) to different extent, that is some animals could depend their choices more heavily on values and some animals could be more dependent on choice preference. In this model, the probability of choosing arm $k$ on trial $t$ :

$$
p_{t}^{k}=\frac{e^{\left(\eta \beta Q_{t}^{k}+(1-\eta) \beta_{c} C K_{t}^{k}\right)}}{\sum_{j} e^{\left(\eta \beta Q_{t}^{j}+(1-\eta) \beta_{c} C K_{t}^{j}\right)}}
$$

793 informative about current choices as the conditional mutual information between the current

794 choice $(\mathrm{C})$ and the last choice (C_t-1), conditioned on the reward outcome of the last trial (R):

$$
I\left(C_{t} ; C_{t-1} \mid R\right)=\sum_{r \in R} \sum_{c_{t-1} \in C} \sum_{C_{t} \in C} p_{C_{t}, C_{t-1}, R}\left(c_{t}, c_{t-1}, r\right) \log \frac{p_{R}(r) p_{C_{t}, c_{t-1}, R}\left(c_{t}, c_{t-1}, r\right)}{p_{C_{t}, R}\left(c_{t}, r\right) p_{C_{t-1}, R}\left(c_{t-1}, r\right)}
$$




\section{Competing Interests}

799 The authors have declared no competing interests.

\section{Acknowledgements}

801 This work was supported by NIMH R01 MH123661, NIMH P50 MH119569, and NIMH T32

802 training grant MH115886, startup funds from the University of Minnesota (N.M.G.), a Young

803 Investigator Grant from the Brain and Behavior Foundation (R.B.E.), an Unfettered Research

804 Grant from the Mistletoe Foundation (R.B.E.), and Fonds de Recherche du Québec Santé,

805 Chercheur-Boursier Junior 1, \#284309 (R.B.E). Thank you to Briana E. Mork and Lisa S. Curry-

806 Pochy for help improve this manuscript.

807

\section{Authors Contributions}

809 Conceptualization, N.M.G., R.B.E., and C.S.C.; Methodology, C.S.C., R.B.E., and N.M.G.;

810 Investigation, C.S.C. E.K. and A.H.; Writing - Original Draft, C.S.C., R.B.E. and E.K.; Writing-

811 Review \& Editing, C.S.C., R.B.E., E.K., and N.M.G.; Funding Acquisition, R.B.E. and N.M.G.;

812 Supervision, R.B.E. and N.M.G. 


\section{References}

Addicott MA, Baranger DAA, Kozink RV, Smoski MJ, Dichter GS, McClernon FJ. 2012. Smoking withdrawal is associated with increases in brain activation during decision making and reward anticipation: a preliminary study. Psychopharmacology 219:563-573. doi:10.1007/s00213-011-2404-3

Addicott MA, Pearson JM, Schechter JC, Sapyta JJ, Weiss MD, Kollins SH. 2020. Attentiondeficit/hyperactivity disorder and the explore/exploit trade-off. Neuropsychopharmacology. doi:10.1038/s41386-020-00881-8

Addicott MA, Pearson JM, Sweitzer MM, Barack DL, Platt ML. 2017. A Primer on Foraging and the Explore/Exploit Trade-Off for Psychiatry Research. Neuropsychopharmacology 42:1931-1939. doi:10.1038/npp.2017.108

Averbeck B, Costa V, Eisenberg D, Czarapata J, Berman K, Murray E. 2017. 376. Subcortical Contributions to the Explore-Exploit Tradeoff. Biological Psychiatry. doi:10.1016/j.biopsych.2017.02.393

Becker JB. 1999. Gender differences in dopaminergic function in striatum and nucleus accumbens. Pharmacol Biochem Behav 64:803-812. doi:10.1016/s0091-3057(99)00168-9

Beeler JA, Daw N, Frazier CRM, Zhuang X. 2010. Tonic dopamine modulates exploitation of reward learning. Front Behav Neurosci 4:170. doi:10.3389/fnbeh.2010.00170

Beeler JA, Frazier CRM, Zhuang X. 2012. Putting desire on a budget: dopamine and energy expenditure, reconciling reward and resources. Front Integr Neurosci 6:49. doi:10.3389/fnint.2012.00049

Berg HC. 1993. Random Walks in Biology. Princeton University Press.

Bilmes J. 1998. A Gentle Tutorial of the EM Algorithm and its Application to Parameter Estimation for Gaussian Mixture and Hidden Markov Models.

Blanco NJ, Otto AR, Maddox WT, Beevers CG, Love BC. 2013. The influence of depression symptoms on exploratory decision-making. Cognition 129:563-568. doi:10.1016/j.cognition.2013.08.018

Chen CS, Becket Ebitz R, Bindas SR, David Redish A, Hayden BY, Grissom NM. 2020. Divergent Strategies for Learning in Males and Females. Curr Biol $\mathbf{0 .}$ doi:10.1016/j.cub.2020.09.075

Cinotti F, Fresno V, Aklil N, Coutureau E, Girard B, Marchand AR, Khamassi M. 2019. Dopamine blockade impairs the exploration-exploitation trade-off in rats. Sci Rep 9:6770. doi:10.1038/s41598-019-43245-Z

Daw ND, O’Doherty JP, Dayan P, Seymour B, Dolan RJ. 2006. Cortical substrates for exploratory decisions in humans. Nature 441:876-879. doi:10.1038/nature04766

Doya K. 2002. Metalearning and neuromodulation. Neural Netw 15:495-506. doi:10.1016/s0893-6080(02)00044-8

Ebitz RB, Albarran E, Moore T. 2018. Exploration Disrupts Choice-Predictive Signals and Alters Dynamics in Prefrontal Cortex. Neuron 97:475. doi:10.1016/j.neuron.2018.01.011

Ebitz RB, Sleezer BJ, Jedema HP, Bradberry CW, Hayden BY. 2019. Tonic exploration governs both flexibility and lapses. PLoS Comput Biol 15:e1007475. doi:10.1371/journal.pcbi.1007475

Ebitz RB, Tu JC, Hayden BY. 2020. Rules warp feature encoding in decision-making circuits. PLoS Biol 18:e3000951. doi:10.1371/journal.pbio.3000951

Filipowicz ALS, Levine J, Piasini E, Tavoni G, Kable JW, Gold JI. 2019. The complexity of model-free and model-based learning strategies. doi:10.1101/2019.12.28.879965 
Frank MJ, Doll BB, Oas-Terpstra J, Moreno F. 2009. Prefrontal and striatal dopaminergic genes predict individual differences in exploration and exploitation. Nat Neurosci 12:1062-1068. doi: $10.1038 / \mathrm{nn} .2342$

Green T, Flash S, Reiss AL. 2019. Sex differences in psychiatric disorders: what we can learn from sex chromosome aneuploidies. Neuropsychopharmacology 44:9-21. doi:10.1038/s41386-018-0153-2

Grissom NM, Reyes TM. 2018. Let's call the whole thing off: evaluating gender and sex differences in executive function. Neuropsychopharmacology. doi:10.1038/s41386-0180179-5

Heath CJ, Phillips BU, Bussey TJ, Saksida LM. 2016. Measuring Motivation and RewardRelated Decision Making in the Rodent Operant Touchscreen System. Curr Protoc Neurosci 74:8.34.1-20. doi:10.1002/0471142301.ns0834s74

Humphries MD, Khamassi M, Gurney K. 2012. Dopaminergic Control of the ExplorationExploitation Trade-Off via the Basal Ganglia. Front Neurosci 6:9. doi:10.3389/fnins.2012.00009

Ishii S, Yoshida W, Yoshimoto J. 2002. Control of exploitation-exploration meta-parameter in reinforcement learning. Neural Netw 15:665-687. doi:10.1016/s0893-6080(02)00056-4

Jenni NL, Larkin JD, Floresco SB. 2017. Prefrontal Dopamine D1 and D2 Receptors Regulate Dissociable Aspects of Decision Making via Distinct Ventral Striatal and Amygdalar Circuits. J Neurosci 37:6200-6213. doi:10.1523/JNEUROSCI.0030-17.2017

Jepma M, Nieuwenhuis S. 2011. Pupil Diameter Predicts Changes in the ExplorationExploitation Trade-off: Evidence for the Adaptive Gain Theory. J Cogn Neurosci 23:15871596. doi:10.1162/jocn.2010.21548

Kool W, McGuire JT, Rosen ZB, Botvinick MM. 2010. Decision making and the avoidance of cognitive demand. J Exp Psychol Gen 139:665-682. doi:10.1037/a0020198

Kurdi B, Gershman SJ, Banaji MR. 2019. Model-free and model-based learning processes in the updating of explicit and implicit evaluations. Proc Natl Acad Sci U S A 116:6035-6044. doi:10.1073/pnas.1820238116

Leao D Jr, Fragoso M, Ruffino P. 2004. Regular conditional probability, disintegration of probability and Radon spaces. Proyecciones 23:15-29.

Maney DL. 2016. Perils and pitfalls of reporting sex differences. Philos Trans $R$ Soc Lond B Biol Sci 371:20150119. doi:10.1098/rstb.2015.0119

McLachlan GJ, Peel D. 2004. Finite Mixture Models. John Wiley \& Sons.

Pearson JM, Hayden BY, Raghavachari S, Platt ML. 2009. Neurons in posterior cingulate cortex signal exploratory decisions in a dynamic multioption choice task. Curr Biol 19:1532-1537. doi:10.1016/j.cub.2009.07.048

Pisupati S, Chartarifsky-Lynn L, Khanal A, Churchland AK. 2019. Lapses in perceptual judgments reflect exploration. Cold Spring Harbor Laboratory. doi:10.1101/613828

Poling A, Edwards TL, Weeden M, Foster TM. 2011. The Matching Law. Psychol Rec 61:313322. doi:10.1007/BF03395762

Schweighofer N, Doya K. 2003. Meta-learning in reinforcement learning. Neural Netw 16:5-9. doi:10.1016/s0893-6080(02)00228-9

Shansky RM. 2019. Are hormones a "female problem" for animal research? Science 364:825826. doi:10.1126/science.aaw7570

van den Bos R, Homberg J, de Visser L. 2013. A critical review of sex differences in decisionmaking tasks: Focus on the Iowa Gambling Task. Behavioural Brain Research. 
906

907

908

909

910

911

912

913 doi:10.1016/j.bbr.2012.10.002

Wilson RC, Bonawitz E, Costa VD, Ebitz RB. 2021. Balancing exploration and exploitation with information and randomization. Curr Opin Behav Sci 38:49-56. doi:10.1016/j.cobeha.2020.10.001

Wyner AD. 1978. A definition of conditional mutual information for arbitrary ensembles. Information and Control 38:51-59. doi:10.1016/S0019-9958(78)90026-8

Yoest KE, Cummings JA, Becker JB. 2014. Estradiol, dopamine and motivation. Cent Nerv Syst Agents Med Chem 14:83-89. doi:10.2174/1871524914666141226103135 